\title{
Nasal alar rim redraping method to prevent alar retraction in rhinoplasty for Asian men: A retrospective case series
}

\author{
Jun Ho Choi ${ }^{1 *}$, Hyokyung Yoo ${ }^{2}$, Byung Jun $\mathrm{Kim}^{2}$ \\ ${ }^{1}$ Department of Plastic and Reconstructive Surgery, Armed Forces Capital Hospital, Seongnam; ${ }^{2}$ Department of Plastic and Reconstructive \\ Surgery, Seoul National University Hospital, Seoul, Korea
}

Background For an attractive and natural tip contour in Asian rhinoplasty, insertion of a nasal implant and reinforcement of the cartilaginous framework are essential. However, scar contracture, which often results from augmentation with implant insertion and inadequate soft tissue coverage of the framework, is one of the most common causes of alar retraction. This study reports a novel method of redraping soft tissue along the alar rim to prevent alar retraction in Asians.

Methods Twenty young Asian men who underwent primary rhinoplasty with septoplasty were retrospectively reviewed. After the usual rhinoplasty procedures, alar rim redraping was conducted for the soft tissue along the transcolumellar and bilateral infracartilaginous incisions. The longest axis of the nostril (a) and the height of the nostril from that axis (b) were measured in anterior-posterior and lateral views. The preoperative and postoperative ratios (b/a) were analyzed using the paired t-test.

Results All 20 patients showed natural contours of the nasal tip, nostrils, and alae after a mean follow-up of 53.6 weeks (range, 52-60 weeks). The ratio of the nostril axes significantly decreased postoperatively in all patients except one, by an average of $11.08 \% \pm 6.52 \%$ in the anterior-posterior view and $17.74 \% \pm 8.49 \%$ in the lateral view $(P<0.01)$. There were no complications, including asymmetry, contracture, subdermal plexus injury, flap congestion, or infection.

Conclusions A quantitative analysis of alar retraction by evaluating the ratio of nostril axes showed that alar rim redraping is a simple and effective adjuvant technique for preventing alar retraction in rhinoplasty for young Asian men.

Keywords Ala / Asian / Rhinoplasty
Correspondence: Byung Jun Kim Department of Plastic and Reconstructive Surgery, Seoul National University Hospital, 101 Daehak-ro, Jongno-gu, Seoul 03080, Korea

Tel: +82-2-2072-1978

Fax: +82-2-2072-0274

E-mail: bjkim79@gmail.com

Received: June 21, 2020 • Revised: November 11, 2020 • Accepted: November 13, 2020 pISSN: 2234-6163・elSSN: 2234-6171・https://doi.org/10.5999/aps.2020.01088・ Arch Plast Surg 2021;48:3-9

\section{INTRODUCTION}

Rhinoplasty is one of the most commonly performed cosmetic surgical procedures in Korea [1,2]. Due to some distinctive ana- tomical characteristics of the nose in Asians, different surgical approaches from the conventional methods described in the Western literature are necessary to obtain satisfactory results. In Asians, the skin is thicker and less pliable, and the cartilage 
framework is much smaller and weaker [3-6]. Moreover, Asians have a low-profile, under-projected nasal tip, increased nostril show, and alar flaring, which all give a feminine appearance [7].

Due to these anatomical features, reinforcement of the cartilage framework, as well as dorsal augmentation, is considered an essential step of rhinoplasty in Asians [3,6]. To obtain sufficient nasal tip projection and nasal length at the same time, the septal extension graft $[4,8,9]$, extended spreader graft $[10]$, columellar strut graft $[11,12]$, and derotation graft are often suggested $[13,14]$. However, reinforcing the cartilage framework often induces a relative deficiency of the nasal soft tissue. Furthermore, capsule formation around the nasal implant can aggravate the nasal retraction by creating soft tissue contracture $[10,15,16]$.

Various surgical techniques have been introduced to prevent soft tissue contracture, such as the lateral crural strut graft or alar rim graft to correct alar retraction [17-19] or the alar spreader graft to relieve pinching of the nasal tip [20]. However, these methods require additional autologous grafts, which increase the surgical time and donor site morbidity. In this study, we introduce a very simple adjuvant technique of redraping the alar rim soft tissue to prevent alar retraction in Asian male patients. The outcomes were quantitatively and statistically analyzed.

\section{METHODS}

\section{Study design}

A retrospective review was performed of patients who underwent primary rhinoplasty with septoplasty between May 2016 and April 2017 at an army hospital. Cases of augmentation rhinoplasty without septoplasty, patients treated with an alar spreader graft, patients with less than 1 year of postoperative follow-up, and revisional procedures were excluded. Preoperative computed tomography was performed in all patients to identify bony asymmetry and septal deviation. Every surgical procedure was performed in accordance with the ethical principles for medical research involving human subjects outlined in the Helsinki Declaration. Verbal and written informed consent and institutional review board approval were obtained for all participants.

\section{Operative technique}

Every operation was performed under general anesthesia. An inverted-V transcolumellar incision with bilateral infracartilaginous endonasal extension was made, and dissection was performed thoroughly, releasing up to the scroll ligament as well as

Table 1. Patient summary and surgical information

\begin{tabular}{|c|c|c|c|c|c|c|c|c|c|}
\hline \multirow[b]{2}{*}{ Patient $^{\text {a) }}$} & \multirow{2}{*}{$\begin{array}{l}\text { Age } \\
(\mathrm{yr})\end{array}$} & \multirow{2}{*}{$\begin{array}{l}\text { Follow-up } \\
\text { period (wk) }\end{array}$} & \multirow{2}{*}{$\begin{array}{l}\text { Trauma } \\
\text { history }\end{array}$} & \multicolumn{2}{|c|}{ Preoperative diagnosis } & \multicolumn{4}{|c|}{ Operative method } \\
\hline & & & & $\begin{array}{l}\text { External } \\
\text { deformity }\end{array}$ & $\begin{array}{c}\text { Septum } \\
\text { deviation }\end{array}$ & Rhinoplasty & $\begin{array}{c}\text { Nasal } \\
\text { implant }\end{array}$ & Septoplasty & $\begin{array}{c}\text { Autologous } \\
\text { cartilage donor site }\end{array}$ \\
\hline 1 & 26 & 54 & None & A & C & ESG, CSG, T & $M$ & SMR & Septum \\
\hline 2 & 25 & 53 & Present & $\mathrm{A}, \mathrm{H}$ & $C, B$ & ESG, CSG, HR, T & $P$ & SMR, CR & Septum \\
\hline 3 & 34 & 60 & Present & $A, H$ & $C, B$ & ESG, CSG, BG, HR, T & $P$ & SMR, CR & Septum \\
\hline 4 & 22 & 52 & Present & $A, H, S$ & $C, B$ & ESG, CSG, BG, HR, T & $P$ & SMR, EBR & Septum \\
\hline 5 & 23 & 53 & Present & $A, H$ & C & ESG, CSG, HR, T & $P$ & SMR & Septum \\
\hline 6 & 21 & 54 & None & $\mathrm{H}$ & None & SEG, HR, T & M & None & Concha \\
\hline 7 & 25 & 53 & Present & $A, H$ & $C, B$ & ESG, CSG, HR, T & $\mathrm{P}$ & SMR, CR & Septum \\
\hline 8 & 24 & 54 & Present & $A, H$ & $C, B$ & ESG, CSG, HR, T & M & SMR, CR & Septum, Concha \\
\hline 9 & 22 & 55 & Present & $A, H$ & $C, B$ & ESG, CSG, HR, T & $P$ & SMR, CR & Septum \\
\hline 10 & 23 & 52 & None & A & C & ESG, CSG, T & M & SMR & Septum \\
\hline 11 & 23 & 53 & Present & $\mathrm{H}$ & C & SEG, HR, T & M & SMR & Septum \\
\hline 12 & 24 & 54 & Present & $\mathrm{A}, \mathrm{H}$ & $C, B$ & ESG, CSG, HR, T & $P$ & SMR, CR & Septum \\
\hline 13 & 22 & 55 & Present & $\mathrm{A}, \mathrm{H}$ & $C, B$ & ESG, CSG, HR, T & $\mathrm{P}$ & SMR, CR & Septum \\
\hline 14 & 25 & 53 & None & $\mathrm{H}$ & None & SEG, HR, T & $P$ & None & Concha \\
\hline 15 & 23 & 52 & None & $A$ & C & $\mathrm{ESG}, \mathrm{CSG}, \mathrm{T}$ & $P$ & SMR & Septum \\
\hline 16 & 22 & 53 & Present & $\mathrm{H}$ & C & SEG, HR, T & M & SMR & Septum \\
\hline 17 & 24 & 53 & Present & $\mathrm{A}, \mathrm{H}$ & $C, B$ & $\mathrm{ESG}, \mathrm{CSG}, \mathrm{HR}, \mathrm{T}$ & $P$ & SMR, CR & Septum \\
\hline 18 & 28 & 54 & Present & $\mathrm{A}, \mathrm{H}$ & $C, B$ & ESG, CSG, BG, HR, T & $P$ & SMR, EBR & Septum \\
\hline 19 & 26 & 53 & Present & $\mathrm{A}, \mathrm{H}, \mathrm{S}$ & $C, B$ & $\mathrm{ESG}, \mathrm{CSG}, \mathrm{BG}, \mathrm{HR}, \mathrm{T}$ & $\mathrm{P}$ & SMR, CR & Septum \\
\hline 20 & 22 & 52 & Present & $\mathrm{H}$ & $C$ & SEG, CSG, HR, T & $P$ & SMR & Septum \\
\hline Mean \pm SD & $24.2 \pm 2$ & $2.9 \quad 53.6 \pm 1.8$ & & & & & & & \\
\hline
\end{tabular}

A, axial deviation; H, hump deformity; S, saddle deformity; C, cartilage; B, bone; ESG, extended spreader graft; CSG, columellar strut graft; T, tip onlay graft; HR, hump reduction; BG, batten graft to upper lateral cartilage; SEG, septal extension graft; M, MegaDerm; P, PureForm; SMR, submucosal resection of septal cartilage; CR, closed reduction of bony septum; EBR, ethmoid bone removal.

${ }^{a}$ All patients are male. 
the nasal hinge complex.

Low-to-high lateral osteotomy through the intranasal approach was performed in 15 patients (75.0\%), and reduction of the dorsal bony hump was performed in 17 patients $(85.0 \%)$ in order to create an even surface for implant insertion. Dorsal augmentation was done in all patients by inserting a subperiosteal implant with the acellular dermal matrix (MegaDerm; L\&C BIO, Seongnam, Korea) in six patients $(30.0 \%)$ and expanded polytetrafluoroethylene (PureForm; Surgiform Technology, Columbia, SC, USA) in 14 patients (70.0\%) (Table 1).

Structural reinforcement was performed by using autologous cartilage, including septal cartilage $(\mathrm{n}=17,85.0 \%)$, conchal cartilage $(n=2,10.0 \%)$, or both $(n=1,5.0 \%)$. In submucosal resection of the septum, the dorsal and caudal $\mathrm{L}$-strut was preserved with a width of at least $12-13 \mathrm{~mm}$ in order to maintain structural stability $[4,9]$. The details of the procedures were different among the patients depending on the surgical indications. In 15 patients with severe axial deviation, an extended spreader graft was performed (75.0\%), while a septal extension graft was used in the other five patients with less severe deviation (25.0\%). A columellar strut graft was fixed at the caudal edge of the cartilaginous septum in 16 patients requiring a more stable tip projection (80.0\%). In four patients with weak side walls, a batten graft was performed on top of the upper lateral cartilage (20.0\%). Finally, a tip onlay graft was done in all patients for prominent tip projection (100\%).

In order to obtain sufficient soft tissue overlying the reinforced framework, we performed nasal alar rim redraping for the bilateral nostrils. With the help of skin flap retraction using a double-

\section{Fig. 1. Incision and range of nasal alar-rim redraping}

Dissection was minimized around the soft triangle area to make subsequent secondary operations possible and was maximized in the lateral areas to obtain sufficient skin flap length.

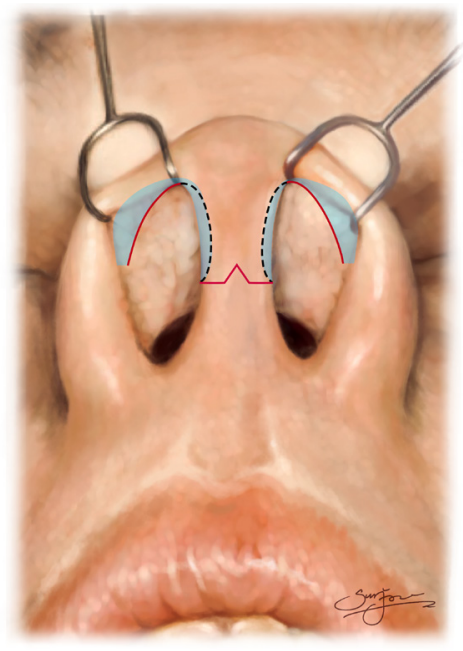

pronged skin hook in one hand, redraping was easily performed along the cephalic margin of the infracartilaginous incisions with the other hand. Dissection was minimized around the soft triangle and maximized in the lateral areas, as shown in Fig. 1, to preserve the soft triangle area as much as possible to make subsequent secondary operations possible. The redraping procedure was performed in the most atraumatic manner possible, and meticulous hemostasis was done to prevent any wound contracture (Fig. 2).

\section{Photographic analysis}

All medical photographs were taken in a single studio by a single photographer under standardized protocols and dimensions. The patient's head was positioned parallel to the Frankfort horizontal plane to maintain a natural head position, and the photographs were taken at the same distance $(1 \mathrm{~m})$. The longest axis of the nostril (a) and the height of the nostril from that axis (b) were measured in anterior-posterior (A-P) and lateral profile views. Then, the preoperative and postoperative ratios of $(\mathrm{b})$ to (a) (b/a), were calculated in order to evaluate the alar-columellar relationship (Fig. 3). Microsoft Paint (Microsoft Corp., Redmond, WA, USA) was used for pixel measurements.

\section{Fig. 2. Schematic drawing of nasal alar rim redraping}

(A) The soft tissue along the infracartilaginous incision. (B) Release of the soft tissue of the nasal alar rim along the incision line with iris scissors. Redraping was performed in the most atraumatic manner possible, and meticulous hemostasis was done to prevent any wound contracture.
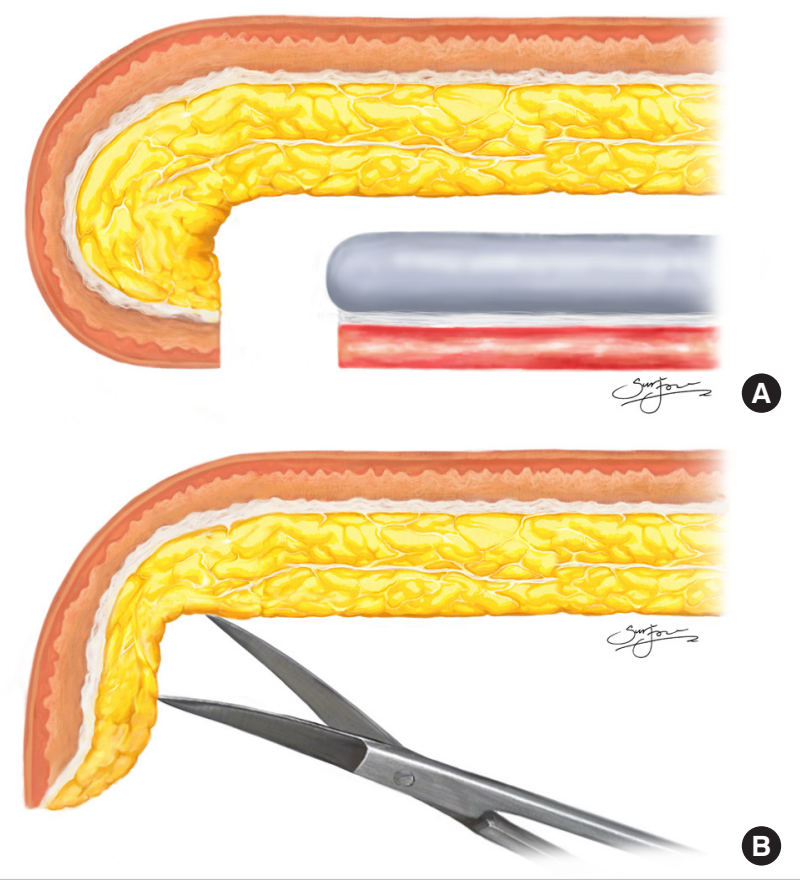


\section{Fig. 3. Quantitative measurements of the nostrils}

The longest axis of the nostril (a) and the height of the nostril from that axis (b) were measured. (A) The anterior-posterior view. (B) The lateral profile view.
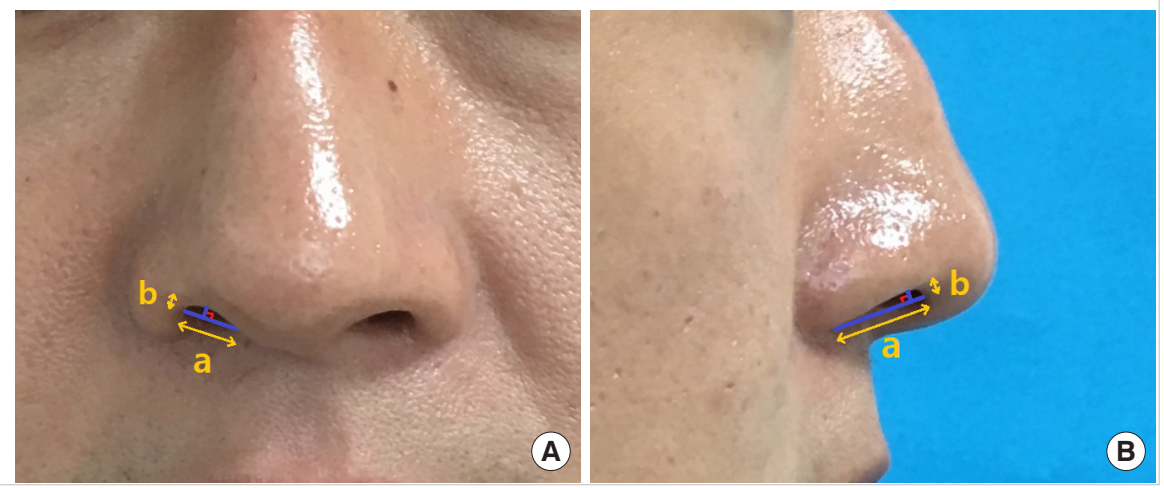

\section{Fig. 4. Preoperative and postoperative photographs of patient 1}

A 26-year-old man with a history of nasal bone fracture received rhinoplasty with dorsal augmentation using an acellular dermal matrix, extended spread graft, columellar strut graft, tip onlay graft, and nasal alar rim redraping. (A) A preoperative photograph in the anterior-posterior (A-P) view. (B) A preoperative photograph in the lateral profile view. (C) A postoperative photograph after 13 months in the A-P view. (D) A postoperative photograph after 13 months in the lateral profile view.
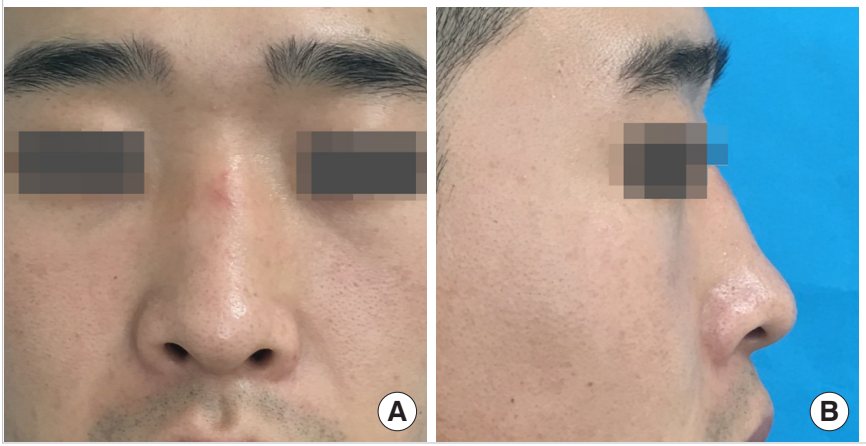
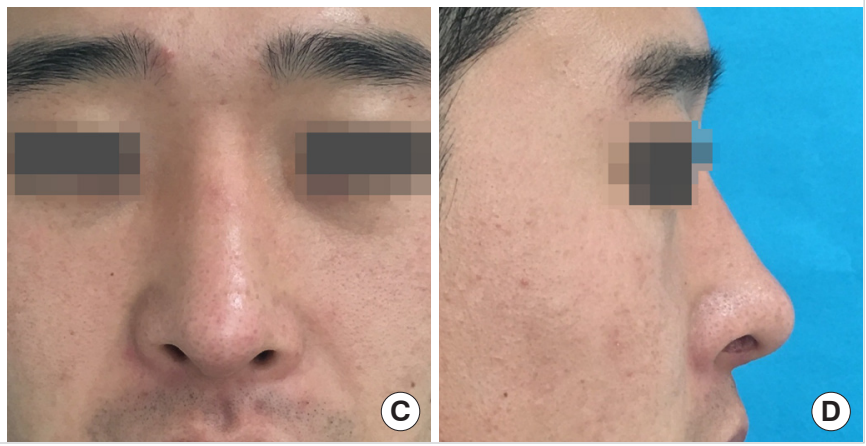

\section{Statistical analysis}

The statistical analysis was performed using SPSS version 23.0 (IBM Corp., Armonk, NY, USA). We used the paired t-test, with P-values less than 0.05 considered to indicate statistical significance, to evaluate the differences between the preoperative and postoperative ratios.

\section{RESULTS}

All 20 patients were young men, with a mean age of 24.2 years (range, 21-34 years), because every patient was treated at an army hospital. Seventeen patients had a traumatic history of nasal bone fracture (77.27\%) and 18 patients had a congenital deformity involving a minor dorsal hump $(90.00 \%)$. The mean follow-up period was 53.6 weeks (range, 52-60 weeks) (Table 1).

All patients showed natural soft tissue contours of the nasal tip, nostrils, and alae after the operation (Figs. 4, 5). The average preoperative ratio of the nostril axes was $30.61 \% \pm 4.05 \%$ in the A-P view and $20.60 \% \pm 4.94 \%$ in the lateral view, whereas the postoperative ratio was $27.19 \% \pm 3.87 \%$ in the A-P view and
$16.92 \pm 4.24 \%$ in the lateral view. The postoperative ratio significantly decreased compared to the preoperative ratio in all patients by an average of $11.08 \% \pm 6.52 \%$ in the A-P view and $17.74 \% \pm 8.49 \%$ in the lateral view $(\mathrm{P}<0.001)$. There was an exception of one case (patient 8 in Table 1), in which the ratio increased by $7.57 \%$ in the A-P view and $9.13 \%$ in the lateral view.

\section{DISCUSSION}

In Asian rhinoplasty, dorsal augmentation and reinforcement of the cartilage framework are among the most essential procedural steps to achieve successful tip refinement. However, creating a stable skeletal framework that is also aesthetically pleasing can be challenging because of the deficiency of soft tissue for coverage and the insidious forces of wound contracture $[13,15]$. These chronic forces of wound contracture often result in alar retraction, which leads to patient dissatisfaction and revision procedures [16,21-23].

Various surgical techniques have been introduced to overcome 


\section{Fig. 5. Preoperative and postoperative photographs of patient 3}

A 34-year-old male patient received rhinoplasty with dorsal hump reduction, augmentation with a PureForm implant, extended spreader graft, columellar strut graft, batten graft, and alar rim redraping. (A) A preoperative photograph in the anterior-posterior (A-P) view. (B) A preoperative photograph in the lateral profile view. (C) A postoperative photograph after 13 months in the A-P view. (D) A postoperative photograph after 13 months in the lateral profile view.
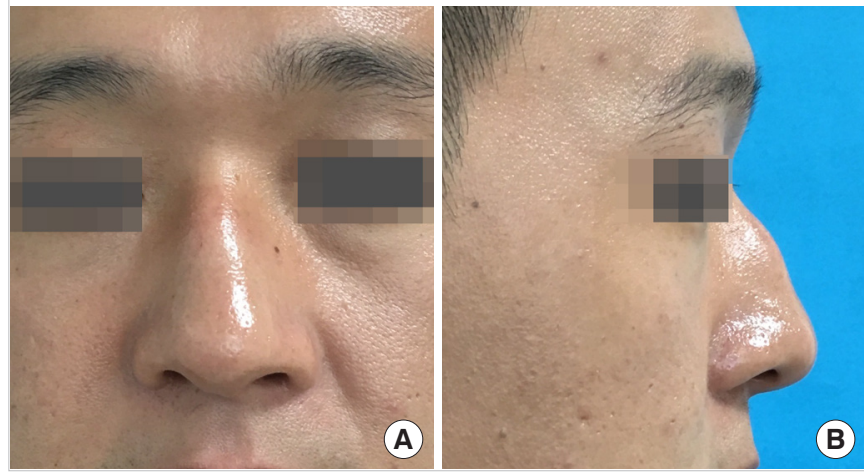

this problem. The lateral crural strut graft was described to correct cephalic malpositioning of the lateral crura by repositioning them in a more caudal orientation $[17,18]$. The direct alar rim graft was also proposed to correct alar retraction [19]. Gunter and Rohrich [20] suggested using the alar spreader graft to relieve pinching of the nasal tip. In Korea, Lee et al. [21] introduced the rib costochondral onlay graft to reinforce the framework and a gull-wing shaped chondrocutaneous graft to resolve the soft tissue deficiency of the middle and inner layers. However, the survival rate of the auricular composite graft was not predictable [24].

These surgical methods to prevent alar retraction all require considerable extra operation time and sufficient amount of additional autologous cartilage, which Asians usually lack. In the present study, we focused on the deficiency of soft tissue, and applied a simple and less time-consuming, but still very effective method that does not require any additional donor morbidity. With help of a skin hook and iris scissors, a single surgeon was able to perform the procedure easily without any assistance. The soft tissue along the cephalic margin of the transcolumellar and infracartilaginous incision was released and expanded by spreading with iris scissors. This reduced skin tension around the wound closure area and eventually led to a more natural contour of the alar rims and nostrils.

The postoperative outcomes were analyzed both subjectively and quantitatively by measuring and statistically analyzing the ratio of nostril axes. The first systematic analysis of the alar-columellar relationship was described by Gunter et al. [25], who measured the absolute distance from the long axis to the superior and inferior borders of the nostrils. However, in this study, the ratio of nostril axes, rather than absolute values, was evaluated to enhance the accuracy of the diagnosis and correction of
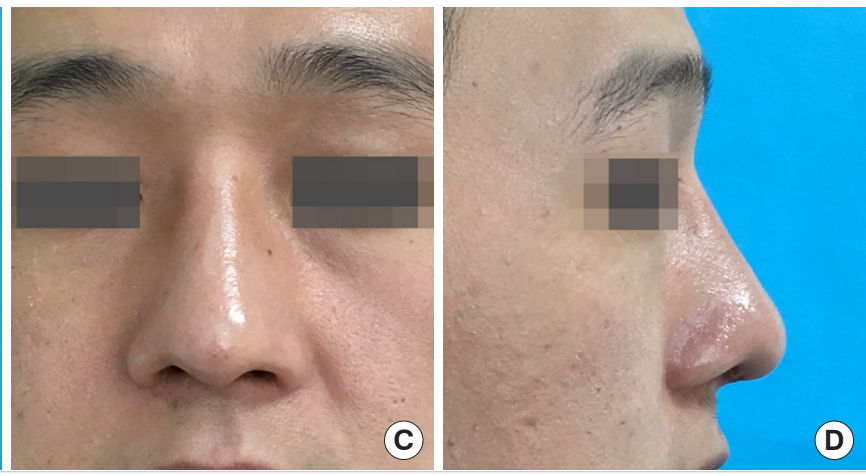

alar retraction. The statistically significant decrease in the ratio of nostril axes in most of the patients (95.0\%) proves the efficacy of this method.

The shape of the alae and nostrils remained natural after the operation without alar retraction. Moreover, there were no complications related to the additional dissection of soft tissue around the incision margins, such as a subdermal plexus injury, nasal flap congestion, hypertrophic scar, or contracted nose. Releasing the connective tissue around the incision line does not seem to have critical effects on the vascularity of the area.

However, our study has some limitations. First, because of the absence of a control group due to the nature of retrospective studies, we compared the postoperative ratio of nostril axes to the preoperative ratio of the same measurement. Moreover, the study size was small and limited to only young men, because every case was performed at an army hospital. Further studies are needed to verify the effectiveness of alar rim redraping in a wider population of patients in terms of age, sex, and race.

In addition, only primary cases were included in this study. For secondary procedures with severe alar retraction, a more extensive range of alar rim redraping should be performed following reinforcement of the skeletal framework. However, dissection should always be as atraumatic as possible and especially minimal and cautious around the soft triangle area. Supplementation with additional soft tissue, such as an auricular composite graft, may be needed in the most severe cases.

Although all photographs were taken in a single studio by a single photographer under standardized protocols, with the patient's head maintained in a natural position, the reproducibility of the medical photographs may have still been limited. To minimize the possible error from inaccuracies in the magnification ratio, the ratios between nostril measurements were analyzed, 
rather than absolute values. Finally, no specific tool was used for the subjective evaluation of postoperative results. Further studies with patient-reported outcomes are needed to support our conclusion.

Despite these limitations, this study is one of the first to describe a quantitative analysis of nasal alar retraction based on the ratio of nostril axes, which enables greater precision in diagnosis and treatment. With the advantage of easy accessibility and minimal donor morbidity, nasal alar rim redraping can be effective for preventing alar retraction in rhinoplasty in Asian men.

\section{NOTES}

\section{Conflict of interest}

No potential conflict of interest relevant to this article was reported.

\section{Ethical approval}

The study was approved by the Institutional Review Board of Seoul National University Hospital (IRB No. 1803-087-930) and performed in accordance with the principles of the Declaration of Helsinki. Verbal and written informed consent was obtained.

\section{Patient consent}

The patients provided written informed consent for the publication and the use of their images.

\section{Author contribution}

Conceptualization: BJ Kim. Data curation: JH Choi. Formal analysis: JH Choi. Project administration: BJ Kim. Visualization: JH Choi. Writing - original draft: H Yoo. Writing - review \& editing: $\mathrm{H}$ Yoo.

\section{ORCID}

Jun Ho Choi https://orcid.org/0000-0001-9762-9929

Hyokyung Yoo https://orcid.org/0000-0002-9171-0379

Byung Jun Kim

\section{REFERENCES}

1. Yang CE, Kim SJ, Kim JH, et al. Usefulness of cross-linked human acellular dermal matrix as an implant for dorsal augmentation in rhinoplasty. Aesthetic Plast Surg 2018;42:28894.

2. Hong JP, Yoon JY, Choi JW. Are polytetrafluoroethylene (Gore-Tex) implants an alternative material for nasal dorsal augmentation in Asians? J Craniofac Surg 2010;21:1750-4.

3. Kim H, Han K. Asian rhinoplasty: correction of the short nose with a septal integration graft. Semin Plast Surg 2015; 29:269-77.

4. Lee SH, Koo MG, Kang ET. Septal cartilage/ethmoid bone composite graft: a new and improved method for the correction underdeveloped nasal septum in patients with short noses. Aesthetic Plast Surg 2017;41:388-94.

5. Kim EK, Daniel RK. Operative techniques in Asian rhinoplasty. Aesthet Surg J 2012;32:1018-30.

6. Han SE, Han K, Choi J, et al. Modified direct-type septal extension grafts: their stability and usefulness in Asian rhinoplasty. Ann Plast Surg 2017;78:243-8.

7. Lam SM. Aesthetic facial surgery for the Asian male. Facial Plast Surg 2005;21:317-23.

8. Gunter JP, Rohrich RJ. Lengthening the aesthetically short nose. Plast Reconstr Surg 1989;83:793-800.

9. Scattolin A, Galzignato PF, Longari F, et al. Septal extension graft in "closed" revision rhinoplasty: a simplified technique. Am J Rhinol Allergy 2017;31:260-4.

10. Lan MY, Jang YJ. Revision rhinoplasty for short noses in the Asian population. JAMA Facial Plast Surg 2015;17:325-32.

11. Rohrich RJ, Hoxworth RE, Kurkjian TJ. The role of the columellar strut in rhinoplasty: indications and rationale. Plast Reconstr Surg 2012;129:118e-125e.

12. Rohrich RJ, Kurkjian TJ, Hoxworth RE, et al. The effect of the columellar strut graft on nasal tip position in primary rhinoplasty. Plast Reconstr Surg 2012;130:926-32.

13. Paik MH, Chu LS. Correction of short nose deformity using a septal extension graft combined with a derotation graft. Arch Plast Surg 2014;41:12-8.

14. Gruber RP. Surgical correction of the short nose. Aesthetic Plast Surg 2002;26 Suppl 1:S6.

15. Park JH, Mangoba DC, Mun SJ, et al. Lengthening the short nose in Asians: key maneuvers and surgical results. JAMA Facial Plast Surg 2013;15:439-47.

16. Kim JH, Park SW, Oh WS, et al. New classification for correction of alar retraction using the alar spreader graft. Aesthetic Plast Surg 2012;36:832-41.

17. Hamilton GS 3rd. Form and function of the nasal tip: reorienting and reshaping the lateral crus. Facial Plast Surg 2016; 32:49-58.

18. Gunter JP, Friedman RM. Lateral crural strut graft: technique and clinical applications in rhinoplasty. Plast Reconstr Surg 1997;99:943-52.

19. Boahene KD, Hilger PA. Alar rim grafting in rhinoplasty: indications, technique, and outcomes. Arch Facial Plast Surg 2009; 11:285-9.

20. Gunter JP, Rohrich RJ. Correction of the pinched nasal tip 
with alar spreader grafts. Plast Reconstr Surg 1992;90:8219.

21. Lee Y, Kim J, Lee E. Lengthening of the postoperative short nose: combined use of a gull-wing concha composite graft and a rib costochondral dorsal onlay graft. Plast Reconstr Surg 2000;105:2190-9.

22. Kim SK, Kim HS. Secondary Asian rhinoplasty: lengthening the short nose. Aesthet Surg J 2013;33:353-62.

23. Kao WTK, Davis RE. Postsurgical alar retraction: etiology and treatment. Facial Plast Surg Clin North Am 2019;27: 491-504.

24. Harbison JM, Kriet JD, Humphrey CD. Improving outcomes for composite grafts in nasal reconstruction. Curr Opin Otolaryngol Head Neck Surg 2012;20:267-73.

25. Gunter JP, Rohrich RJ, Friedman RM. Classification and correction of alar-columellar discrepancies in rhinoplasty. Plast Reconstr Surg 1996;97:643-8. 